\title{
A CHARACTERIZATION THEOREM FOR CELLULAR MAPS
}

\author{
BY WILLIAM HAVER ${ }^{1}$
}

Communicated by Steve Armentrout, March 26, 1970

Introduction. The main result of this paper is that a mapping $f$ of the $n$-sphere $\partial B^{n+1}, n \neq 4$, onto itself is cellular if and only if $f$ has a continuous extension which maps the interior of the $n+1$ ball $B^{n+1}$ homeomorphically onto itself. Since a map of a 2-sphere onto itself is cellular if and only if it is monotone, this theorem extends a result of Floyd and Fort [6], who prove the corresponding theorem for monotone maps on a 2 -sphere.

Preliminaries. A compact mapping $f: M^{n} \rightarrow X$ is cellular if for each $x \in X$, there is a sequence $C_{1}, C_{2}, \cdots$ of topological $n$-cells such that $f^{-1}(x)=\bigcap_{i=1}^{\infty} C_{i}$ and $C_{i+1} \subset \operatorname{Int} C_{i}$. If $X$ is a topological space, $H(X)$ is the group of all homeomorphisms of $X$ onto itself. Edwards and Kirby showed that for any compact manifold $M, H(M)$ is locally contractible and therefore uniformly locally arcwise connected. It was shown [7] that any mapping of a manifold onto itself which can be uniformly approximated by homeomorphisms is cellular. (See also [4].) Armentrout $(n=3)$ [1] and Siebenmann $(n \geqq 5)$ [10] have proven that any cellular mapping of a manifold onto itself can be uniformly approximated by homeomorphisms.

Lemma 1. Suppose $f: \partial B^{n} \rightarrow \partial B^{n}$ can be approximated by homeomorphisms. Then $f$ can be extended to a map which is a homeomorphism on the interior of $B^{n}$.

Proof. Since $f$ can be uniformly approximated by homeomorphisms and $H\left(\partial B^{n}\right)$ is uniformly arcwise connected, there is an arc $\Phi$ such that $\Phi_{1}=f$ and $\Phi_{t} \in H\left(\partial B^{n}\right)$, for $0 \leqq t<1$. Each point of $B^{n}$ can be represented in the form $t x$, where $x \in \partial B^{n}$ and $0=t=1$. We define $F: B^{n} \rightarrow B^{n}$ by $F(t x)=t \Phi_{t}(x)$, for all $x \in \partial B^{n}$. We note that $F$ is continuous, extends $f$ and is a homeomorphism when restricted to the interior of $B^{n}$.

Therefore, if $n \neq 4$ and $f: \partial B^{n+1} \rightarrow \partial B^{n+1}$ is cellular $f$ can be extended to a map which is a homeomorphism on the interior of $B^{n+1}$.

AMS 1970 subject classifications. Primary 5460; Secondary 5701.

Key words and phrases. Cellular maps, monotone maps, extending mappings, $\mathrm{UV}^{\infty}$ maps.

1 This paper represents a portion of the author's Ph.D. thesis, written under the direction of Louis F. McAuley to be presented to the faculty of the State University of New York at Binghamton. 
A map has property $U V^{\infty}$ if for each $x$ and each open set $U$ containing $f^{-1}(x)$, there is an open set $V$ containing $f^{-1}(x)$ such that $V \subset U$ and $V$ is null-homotopic in $U$.

Lemma 2. Let $M$ be a manifold and $F: M \times(0,1] \rightarrow M \times(0,1]$ be $a$ map such that $F^{-1}(M \times 1)=M \times 1$ and $F / M \times(0,1): M \times(0,1) \rightarrow M$ $\times(0,1)$ is a homeomorphism, then $F / M \times 1: M \times 1 \rightarrow M \times 1$ is a $\mathrm{UV}^{\infty}$ map.

Proof. We identify $M$ with $M \times 1$. We make use of the following auxiliary maps: for each $\partial$, define $\pi_{\partial}: M \rightarrow M \times(1-\partial)$ by $\pi_{\partial}(x)$ $=(x, 1-\partial)$ and $p: M \times(0,1] \rightarrow M$ by $p(x, t)=(x, 1)=x$.

Let $U^{\prime}$ be open in $M$ with $f^{-1}(b) \subset U^{\prime} . U^{\prime} \times(0,1]$ is open in $M$ $\times(0,1]$. Therefore, there is a $U$ such that:

(a) $U$ is open in $M \times(0,1]$.

(b) $U \subset U^{\prime} \times(0,1]$.

(c) $f(U)$ is open in $M \times(0,1]$.

(d) $F^{-1}(b) \subset U$.

Now choose $t_{0}<1$ and an open cylinder, $C$, about $b \times\left[t_{0}, 1\right]$ such that $C \subset f(U)$. We note that:

$f^{-1}(C)$ is open in $M \times(0,1], f^{-1}(C) \subset U, f^{-1}\left(b \times\left[t_{0}, 1\right]\right) \subset f^{-1}(C)$. Let $\eta=d(b, \widetilde{C}) ; \eta>0$. Let $\delta$ be chosen so that

(a) $N_{2 \delta}\left(f^{-1}(b)\right) \subset f^{-1}(C)$.

(b) $d(x, y)<2 \delta \Rightarrow d(f(x), f(y))<\eta$.

Let $V=N_{\delta}\left(f^{-1}(b)\right) \cap M$. We note that if $x$ is an element of $\pi_{\delta}(V)$, then $f(x)$ is an element of $N_{\eta}(b) \cap M \times(0,1) \subset C$.

Since $C$ is a cell we can define a homotopy $G: C \times I \rightarrow C$ so that

(1) $x \in C^{\circ} \Rightarrow G(x, t) \in C \cap(M \times(0,1))$.

(2) $G(x, 0)=x$.

(3) $\exists z \in M \times(0,1)$ such that $G(x, 1)=z$, for all $x \in C$.

We now can define the desired homotopy $H: V \times I \rightarrow U^{\prime}$, by $H(x, t)$ $=p f^{-1}\left(G\left(f \pi_{\delta}(x), t\right)\right)$. Thus, $H(x, 0)=p f^{-1}\left[G\left(f \pi_{\delta}(x), 0\right)\right]=p f^{-1}\left(f \pi_{\delta}(x)\right)$ $=x$.

$$
H(x, 1)=p f^{-1}\left[G\left(f \pi_{\delta}(x), 1\right)\right]=p f^{-1}(z)=\text { constant. }
$$

The continuity of $f$ follows from that of $G$, so all that remains to be shown is that $H(x, t) \in U^{\prime}$, for all $x \in V, \forall t \in I$.

$$
\begin{aligned}
x \in V & \Rightarrow \pi_{\delta}(x) \in \pi_{\delta}(V) \Rightarrow f\left(\pi_{\delta}(x)\right) \in C \cap M \times(0,1) \\
& \Rightarrow G\left(f \pi_{\delta}(x), t\right) \in C \cap B^{\circ} \Rightarrow
\end{aligned}
$$

that $f^{-1}$ is defined and $f^{-1}\left[G\left(f \pi_{\dot{\delta}}(x), t\right)\right] \in f^{-1}(C) \subset U \subset U^{\prime} \times\left(\frac{1}{2}, 1\right]$. Thus $P\left(f^{-1}\left[G\left(f \pi_{\delta}(x), t\right)\right]\right)=H(x, t) \in U^{\prime}$. 
Let $M \subset X . M$ is collared if there is a homeomorphism $h: M \times(0,1]$ $\rightarrow$ nbd of $M$ such that $h(m, 1)=m$, for all $m \in M$. M. Brown proved that the boundary of any manifold with boundary is collared [3]. Therefore, we have the following corollary.

CoRollary. Let $M$ be a manifold with boundary and let $f: M \rightarrow M$ be such that $f$ restricted to the interior of $M$ is a homeomorphism. Then $f / \partial M$ is a $\mathrm{UV}^{\infty}$-map.

Using McMillan's criteria for cellularity, [9] it can easily be shown that if $f: M^{n} \rightarrow M^{n}$ is a UV'-map and if $M^{n}=S^{3}$ or $n \geqq 5$, then $f$ is a cellular map. (Cf., Armentrout and Price [2] or Lacher [8].) We therefore have the following theorem:

THEOREM. $A$ mapping $f$ of the $n$-sphere $\partial B^{n+1}, n \neq 4$, onto itself is cellular iff $f$ has a continuous extension which maps the interior of $B^{4}$ homeomorphically onto itself.

Corollary. Let $M$ be an m-manifold, $n \geqq 5$, with boundary. Let $f$ be a map of $M$ onto $M$ such that $f /$ Int $M:$ Int $M \rightarrow \operatorname{Int} M$ is cellular and $f / \partial M: \partial M \rightarrow \partial M$. Then $f / \partial M$ is a $\mathrm{UV}^{\infty}$ map. In particular, if $n \geqq 6$, $f / M$ is a cellular map.

Proof. Define $g:$ Int $M \rightarrow(0, \infty)$ by $g(m)=d(m, \partial M)$. Since $f /$ Int $M$ is a cellular map, by Siebenmann's theorem there is a homeomorphism $h$ such that for all $x \in \operatorname{Int} M, d(f(x), h(x))<g(f(x))$. We define $F: M \rightarrow M$ by

$$
\begin{aligned}
F(x) & =h(x), & & x \in \operatorname{Int} M, \\
& =f(x), & & x \in \partial M .
\end{aligned}
$$

$F$ is continuous, for suppose there is a sequence, $x_{n}$, of points in Int $M$ which converge to $x \in \partial M$. Let $\epsilon>0$ be given. By the continuity of $f$, $\exists N \ni n>N \Rightarrow d\left(f_{n}(x), f(x)\right)<\epsilon / 2$. Then for such $n$,

$d\left(F\left(x_{n}\right), F(x)\right)=d\left(h\left(x_{n}\right), f(x)\right) \leqq d\left(h\left(x_{n}\right), f\left(x_{n}\right)\right)+d\left(f\left(x_{n}\right), f(x)\right)<\epsilon$.

Thus, by Lemma $2, F / \partial M=f / \partial M$ is a $U^{\infty}$ map.

Armentrout's approximation theorem [1] and results of E. E. Floyd [5] make it possible to prove the corresponding result for three manifolds: For such $M$, if $f: M \rightarrow M$ is a proper map such that $f / \operatorname{Int} M$ is cellular, then $f / \partial M$ is cellular.

\section{REFERENCES}

1. S. Armentrout, Concerning cellular decompositions of 3-manifolds that yield 3manifolds, Trans. Amer. Math. Soc. 133 (1968), 307-332. MR 37 \#5859. 
2. S. Armentrout and T. Price, Decompositions into compact sets with UV properties, Trans. Amer. Math. Soc. 141 (1969), 433-446.

3. M. Brown, Locally flat imbeddings of topological manifolds, Ann. of Math. (2) 75 (1962), 331-341. MR 24 \#A3637.

4. R. L. Finney, Uniform limits of compact cell-like maps, Notices Amer. Math. Soc. 15 (1968), 942. Abstract \#68T-G26.

5. E. E. Floyd, The extension of homeomorphisms, Duke Math. J. 16 (1949), 225235. MR 10, 726.

6. E. E. Floyd and M. K. Fort, A characterization theorem for monotone mappings, Proc. Amer. Math. Soc. 4 (1953), 828-830. MR 15, 244.

7. W. Haver, Cellular mappings on three manifolds, Notices Amer. Math. Soc. 16 (1969), 1054. Abstract \#669-39.

8. R. C. Lacher, Cellularity criteria for maps (to appear).

9. D. R. McMillan, Jr., A criterion for cellularity in a manifold, Ann. of Math. (2) 79 (1964), 327-337. MR 28 \#4528.

10. Siebenmann, Approximating cellular maps by homeomorphisms (to appear). 13901

State University of New York at Binghamton, Binghamton, New York 